\title{
GhCOR27, Which Encodes a Cold-related Gene, Is Involved in Cotton Tolerance to Cold Stress
}

\author{
Jianping Li ${ }^{1,2}$, Xiaoyan Hao ${ }^{1,2}$, Zumuremu Tuerxun ${ }^{1,2}$, Xiaochun Chang ${ }^{1,2}$, Shengqi Gao ${ }^{1,2}$, \\ Wenran Hu ${ }^{1,2}$, Guo Chen ${ }^{1,2}$, Quansheng Huang ${ }^{1,2, *}$ \\ ${ }^{1}$ Institute of Nuclear Technology and Biotechnology, Xinjiang Academy of Agricultural Sciences, Urumqi, China \\ ${ }^{2}$ Xinjiang Key Laboratory of Crop Biotechnology, Urumqi, China
}

Email address:

hquansheng@126.com (Quansheng Huang)

*Corresponding author

\section{To cite this article:}

Jianping Li, Xiaoyan Hao, Zumuremu Tuerxun, Xiaochun Chang, Shengqi Gao, Wenran Hu, Guo Chen, Quansheng Huang. GhCOR27, Which Encodes a Cold-related Gene, Is Involved in Cotton Tolerance to Cold Stress. International Journal of Applied Agricultural Sciences.

Vol. 6, No. 1, 2020, pp. 1-6. doi: 10.11648/j.ijaas.20200601.11

Received: December 8, 2019; Accepted: December 25, 2019; Published: January 8, 2020

\begin{abstract}
The growth and development of crop plants are greatly affected by various abiotic stresses such as cold stress. Cold stress tolerance is triggered by a sensing mechanism, leading to signaling transduction in plants. Especially in the model species Arabidopsis (Arabidopsis thaliana) it was found that cold-related genes play important roles via cold stress. Cotton is thermophilic and sensitive to cold temperature during its development and growth. However, the mechanism of about cotton how to sense the cold response remains elusive. Here, we isolated a gene from cotton seedling cDNA, which phylogenetic analysis clustered it into the Cold-Related gene member and the protein sequences showed that its closest homolog is Arabidopsis AtCOR27, so we defined it as GhCOR27. Furthermore, the expression pattern analysis via real-time PCR used peculiar GhCOR 27 primers demonstrated that GhCOR 27 mRNA accumulated abundance was gradually induced at low temperature. In addition, the cotton seedlings of silenced-GhCOR27 through virus-induced gene silencing (VIGS) exhibited a more severe chilling injury phenotype at low temperature. These results suggested that GhCOR27 may play a role in response to cotton cold stress response.
\end{abstract}

Keywords: Gossypium hirsutum, Cold Stress, Cold-related Gene (COR), Virus-induced Gene Silencing (VIGS)

\section{Introduction}

Plant suffers various biotic and abiotic factors in its life cycle which affect plant growth and development. Abiotic factors such as drought, temperature and salt can cause irreversible injury to plant leading to poor growth, the loss of yield and even leading to death. For many annual plants such as maize, cotton and rice which are sensitive to temperature, cold stress becomes the primary environmental factor affecting the growth, development and yield [1]. Also, the degree of damages depends on the period of exposure to cold stress and the ability of adaption to cold stress [2]. In order to survive under cold condition, over a long evolutionary period, plants develop the ability to sense low temperature and the mechanisms of stress tolerance [3].

The evolved adaption to cold stress called cold acclimation, is associated with a complex process involved in multiple signaling pathways including biochemical and physiological alterations such as activating second messengers and other components to respond to cold tress. According to previous studies, low temperature activates second messengers $\mathrm{Ca}^{2+}$ to trigger plant responses under cold stress, which cellular $\mathrm{Ca}^{2+}$ dynamics are detected in response to cold through a aequorin-based $\mathrm{Ca}^{2+}$-signaling mechanism [4] and the reduction in membrane fluidity caused by cold stress appears to be a primary event of cold perception to activate the $\mathrm{Ca}^{2+}$ channel in plants [5]. Molecular traits were revealed that many genes are involved in the cold acclimation. A group of genes named cold regulated genes $(C O R)$ genes have been characterized as down-stream functional protein in $C B F$-dependent pathway designed as the ICE1-CBF-COR cascade which were widely studied. In this system, $\mathrm{CBF} / \mathrm{DREBs}$ are rapidly induced by cold, and bind to the 
promoter regions of COR genes to activate their transcription [6]. Furthermore, expression of many $C O R$ genes was induced by cold stress than that of $C B F$ genes [7]. So far, many $C O R$ genes have been isolated from plant species, and reported to play roles in low temperature pathway, especially in Arabidopsis, the COR genes such as COR6.6, COR15a, COR47, and COR27, COR28, COR78, have been well characterized and acknowledged [8-11]. But, bioinformatic analysis indicated that $C O R$ family have lower homology in plants compared with the $C B F$ family [12].

Cotton, as widely planted crop in China, often suffer from low temperature leading to loss of yield and even to death especially in spring in some districts of China such as Xinjiang. Therefore, based on biological and agricultural need, the research and understanding on the cotton cold acclimation mechanism at biochemical and molecular level will provide important help. By now, the knowledges of COR genes in response to cold stress in cotton remain less. Here, we isolated a gene from cotton seedling cDNA. Polygenetic analysis by amino sequence alignment indicated that it belongs to the same clade with AtCOR27, so we defined it as GhCOR27. The expression pattern analysis demonstrated that GhCOR27 was induced and its mRNA accumulated abundance increased at low temperature. Furthermore, the cotton plants of silenced-GhCOR27 through virus-induced gene silencing (VIGS) exhibited a more severe chilling injury phenotype at low temperature. These results suggested that GhCOR27 may play a role in response to cotton cold stress.

\section{Materials and Methods}

\subsection{Cotton Growth and Gene Expression Analysis}

Seeds of upland cotton cv TM-1 (Gossypium hirsutum) were delinted with $\mathrm{H}_{2} \mathrm{SO}_{4}(98 \%)$ and sterilized in $70 \%$ ethanol for a few seconds, followed by three rinses in sterile water. Cotton plants were grown in a controlled environment chamber at $28^{\circ} \mathrm{C}$, with a 12 hours photoperiod. For cold treatments, 3-week-old seedlings were transferred to $10^{\circ} \mathrm{C}$, and were collected at 3, 6, 12, 24 and $48 \mathrm{~h}$ timepoint used for RNA extraction and real-time PCR analysis. Total RNA was isolated from leaf tissue using the Biospin Plant Total RNA Extraction Kit (Bioer Technology Co., Ltd. China) and First-strand cDNA was synthesized by TranScript-Uni One-Step gDNA Removal and cDNA synthesis SuperMix kit (TRANSGEN BIOTECH Co., Ltd. China). For qRT-PCR analysis, the gene-specific primers GhCOR27-F: 5-GGAGAAAAGACCAGTTATGC-3 and GhCOR27-R: 5-TAATGTGTGTCAGACTTCGG-3 and the primers histon3-F: 5-GCCAAGCGTGTCACAATTATGC-3 and histon3-R: 5-ACATCACATTGAACCTACCACTACC-3 were selected and checked via BLASTN search.

\subsection{Phylogenetic Analysis}

The neighbor-joining method was used to construct the phylogenetic tree of using MEGA v. 5.1 [www.megasoftware.net]. Amino acid sequences were analyzed using DNAMAN (DNAMAN Inc). The peptide sequences were aligned with the ClustalW program (http://www.ebi.ac.uk), and phylogenetic analysis was employed to investigate the evolutionary relationships among the aquaporins. A minimum evolution tree was generated in MEGA-X.

\subsection{Construction of Vigs Vectors and Agrobacterium Mediated Vigs}

Tobacco rattle virus (TRV)-based virus-induced gene silencing was performed. GhCOR27 was amplified by polymerase chain reaction from cDNA of TM-1 leaf tissues with primers GhCOR27-F: 5-GAATTCCTTCTCATCAGTTTTCTATCCT-3, GhCOR27-R:

5-GGGGTACCGTAATGTGTGTCAGACTTCGGG-3, and inserted into the pYL156 vector with restriction enzymes EcoRI and KpnI digestion. GhCLAl was cloned into the pYL156 (pTRV-RNA2) vector as control, which is used as a visible marker to monitor the efficiency of VIGS. The plasmids containing binary TRV vectors pTRV-RNA1 and pTRV-RNA2 (pYL156) vector, pYL156-GhCOR27 and pYL156-GhCLA1, were transformed into Agrobacterium tumefaciens strain GV3101, respectively. Ten-d-old seedlings were transfected with the mixture (1:1, v/v) of Agrobacterium cultures $(\mathrm{OD} 600=1.5)$ harboring pTRV1 with pTRV2 or its derivative plasmids. After completion of agro-inoculation, the seedlings were grown at $25^{\circ} \mathrm{C}$ under a $16 \mathrm{~h} / 8 \mathrm{~h}$ light/dark cycle in a controlled environmental chamber. After two weeks of cultivation, the plants were inoculated for two weeks with low temperature. The experiments were performed with at least 30 plants per treatment and repeated three times.

\section{Results}

\subsection{GhCOR27 Bioinformatic and Phylogenetic Analyses}

It remains unknown how many COR family members exist in cotton and what their biological functions are. To identify the genes involved in response to low temperature in cotton, the published amino acid sequences of AtCOR27 genes were retrieved and used as query to blast against the G. hirsutum unigene database (https://www.cottongen.org/). Nine genes from the G. hirsutum unigene database, Gh_A01G0983, Gh_A06G1853, Gh_A09G0755, Gh_D01G0071, Gh_D01G1032, Gh_D05G1942, Gh_D06G0147, Gh_D09G0756 and Gh_D12G0215 were retrieved. We performed the expression analysis under low temperature treatment for all the nine genes (data not shown) and showed Gh_A09G0755 was induced by low temperature. Then, by using online blast tool (https://blast.ncbi.nlm.nih.gov/Blast.cgi), the deduced amino acid sequence of Gh_A09G0755 was used to blast with AtCOR27 (NP 568615.3, Arabidopsis thaliana), PvCOR27 (XP_031267232.1, Pistacia vera), PgCOR27 (XP_031394028.1, Punica granatum), TcCOR27 (EOY14453.1, Theobroma cacao) and GaCOR27 
(KAA3476458.1, Gossypium australe) and exhibited high identity with GaCOR27 (89.86\%), TcCOR27 (74.06\%), but lower identity with PvCOR27 (52.57\%), AtCOR27 (47.86\%) and PgCOR27 (35.23\%) (Figure 1A). Meanwhile alignment was performed using the multalin website (http://multalin.toulouse.inra.fr/multalin/) and shown these COR family member containing common conserved domain, so we designed Gh_A09G0755 as GhCOR27 (Figure 1B).
A

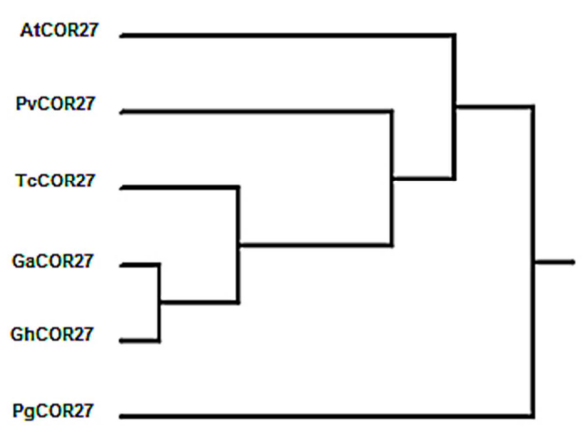

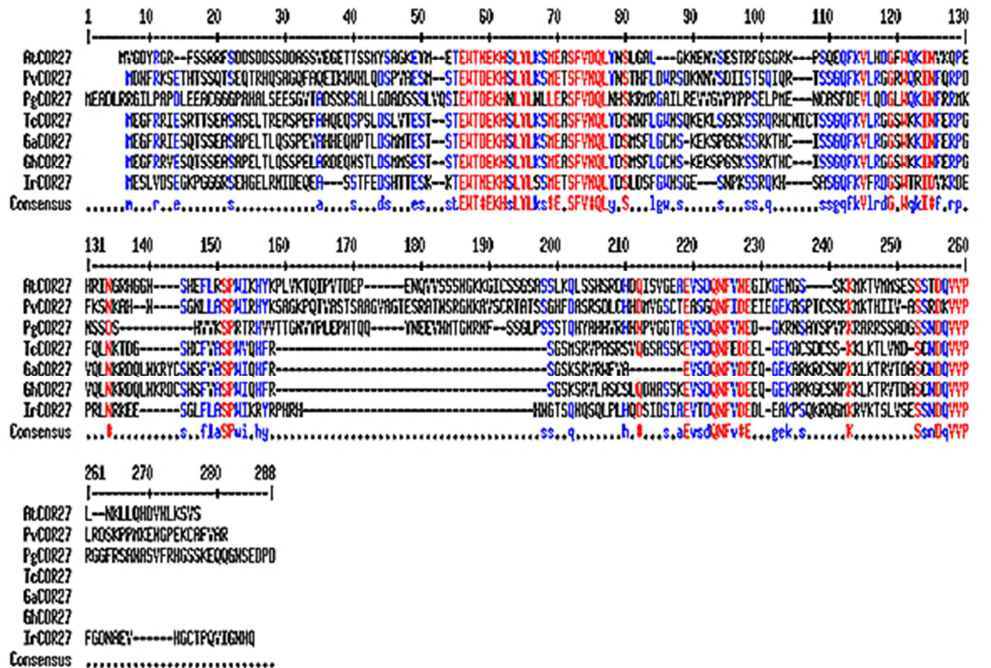

Figure 1. A Sequence alignment and B phylogenetic analysis of cotton GhCOR27 and other species COR27 family members.

The alignment was performed using the Multalin website (http://multalin.toulouse.inra.fr/multalin/) with a hierarchical clustering approach, and the phylogenetic tree was constructed using CLUSTALW and rooted phylogenetic tree (http://www.genome.jp/tools/clustalw/).

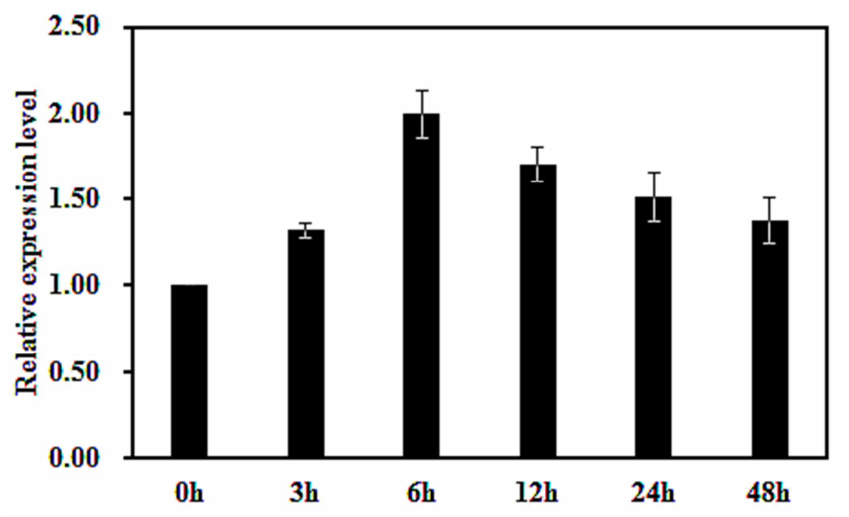

Figure 2. Relative expression analysis of GhCOR27 in cotton under low temperature treatments.

GhCOR27 expression patterns were analyzed in 3-week-old plants growing at $26^{\circ} \mathrm{C}$ after treatments at $10^{\circ} \mathrm{C}$ for $0,3,6,12,24$ and $48 \mathrm{~h}$. The results are the mean value of three independent experiments. For each experiment 25-30 plants were used per treatment. Bars represent SEs, $\mathrm{P}<0.05$.

\subsection{The Expression of GhCOR27 Is Induced by Low Temperature}

To investigate whether GhCOR27 is involved in defense low temperature stress, we checked the expressive pattern of GhCOR 27 at low temperature. the seedlings were shifted from $26^{\circ} \mathrm{C}$ to $10^{\circ} \mathrm{C}$ and then were collected at $3 \mathrm{~h}, 6 \mathrm{~h}, 12 \mathrm{~h}, 24 \mathrm{~h}, 48 \mathrm{~h}$ time points respectively for transcriptional analysis. An induction was observed following treatment at $10^{\circ} \mathrm{C}$, with the increasement of incubation time at low temperature, the transcriptional level of GhCOR27 was gradually induced, a peak expression was showed at 6h-time point (Figure 2). Based on the result, we speculated that GhCOR27 may function in the cold acclimation process in cotton.

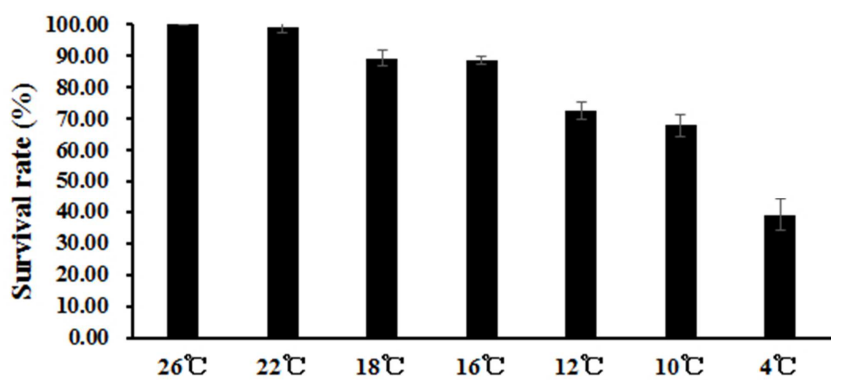

Figure 3. Survival rates of cotton seedlings treated at different temperature.

Plants were grown at $26^{\circ} \mathrm{C}$ for 3 weeks and transferred directly to $22^{\circ} \mathrm{C}, 18^{\circ} \mathrm{C}$, $16^{\circ} \mathrm{C}, 12^{\circ} \mathrm{C}, 10^{\circ} \mathrm{C}$ and $4{ }^{\circ} \mathrm{C}$ for $3 \mathrm{~d}$ in normal photoperiod $(12 \mathrm{~h}$-light $/ 12 \mathrm{~h}$-dark cycle). And then returned to $26^{\circ} \mathrm{C}$ for $72 \mathrm{~h}$. The survival rates were monitored. The results are the mean value of three independent experiments. For each experiment 25-30 plants were used per treatment. Bars represent $\mathrm{SEs}, \mathrm{P}<0.05$.

\subsection{Assessment of Survival Following Low Temperature Treatment in Acclimated and Non-acclimated Plants}

Cotton as a crop sensitive to temperature, its growth development has great chance of suffering from cold stress, Seedlings exposed to cold often produce lower survival [2]. Here, a test based on survival rate to establish proper low temperature at which phenotypic differences between seedlings with gene-overexpressing or gene-silencing and wild-type seedlings can be told clearly was performed. 
3-week-old cotton seedlings growing at $26^{\circ} \mathrm{C}$ were shifted to $22^{\circ} \mathrm{C}, 18^{\circ} \mathrm{C}, 16^{\circ} \mathrm{C}, 12^{\circ} \mathrm{C}, 10^{\circ} \mathrm{C}$ and $4^{\circ} \mathrm{C}$ for 3 days in normal photoperiod (12-h-light/12-h-dark cycle). And the percentage of survival was investigated after transferring back to $26^{\circ} \mathrm{C}$ for $3 \mathrm{~d}$. As shown, with the decrease of temperature, seedlings exhibited increasing sensitivity to low temperature with decreased survival rates, and survival rates reached the lowest especially when seedlings transferred from $26^{\circ} \mathrm{C}$ to $4^{\circ} \mathrm{C}$ (Figure 3). The compared result indicated that temperature begin to show impact on seedlings in survival rate at $18^{\circ} \mathrm{C}$ and use of lower temperature at $4{ }^{\circ} \mathrm{C}$ was lethal for cotton. But for the purpose of telling significant difference between VIGS plants and control for following gene function analysis under certain cold stress condition, the pre-test proved that the better cold stress treatment was at $10^{\circ} \mathrm{C}$.

\subsection{VIGS Gene-silencing Cotton Plants Exhibited Supersensitive to Low Temperature}

Since VIGS gene-silencing technique is a rapid way for studying gene profile in plant and is wildly applied to discovery functional gene in cotton [13-15]. To further test whether GhCOR27 was required for cotton resistance at low temperature, we adopted VIGS assay in this study. First, we infiltrated GhCLA1 into seedling leave as a visual marker to verify the efficiency in TM-1, after 10-15 days, newly

A
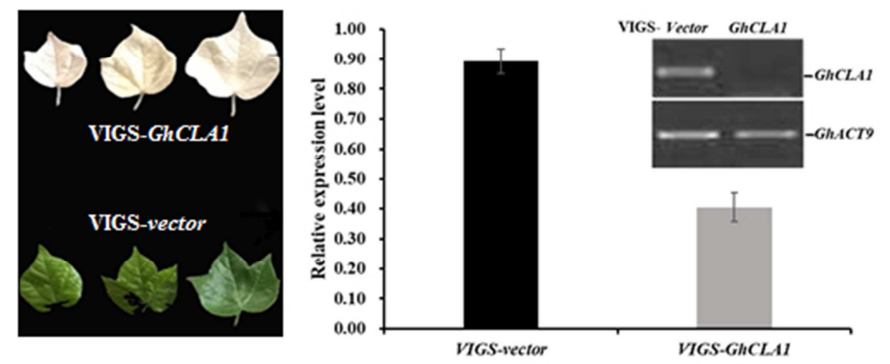

B

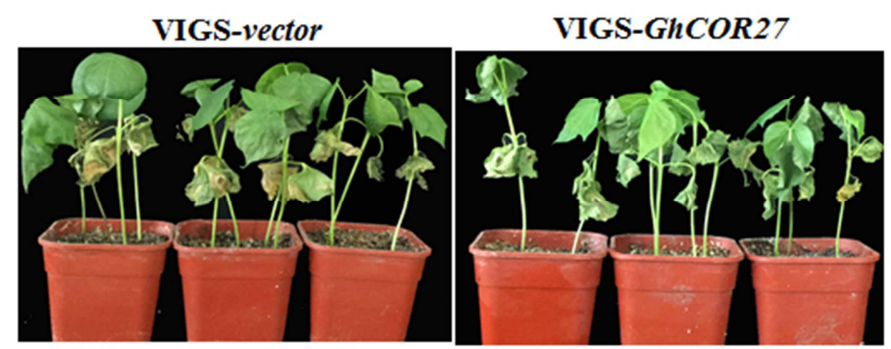

emerged true leave exhibited albino phenotype, Meanwhile decreased transcriptional level for GhCLA1 was also recorded by RT-PCR and qPCR (Figure 4A and 4B). So, the VIGS assay was establish in this study for silencing GhCOR27 in cotton. Then we designed the primers to amplify the conserved region of GhCOR27 and inserted it into TRV-RNA2 vector pYL156. The Agrobacterium carrying the recombinant TRV vectors with either vector control or GhCOR27 fragment were infiltrated into the cotton seedlings, seedlings infiltrated with GhCLA1 were set as marker to assess the silencing efficiency for GhCOR27 silencing in this study. When the marker seedings showed albino phenotype, we challenged the control and GhCOR27, seedlings with low temperature. The phenotype in TM-1 plants silenced with GhCOR 27 by VIGS was observed, the expression of interesting gene was analysis by both RT-PCR and $\mathrm{qPCR}$ (4B and 4C). Also the percentage of survival plants was scored after treatment at low temperature. As shown, plants silenced with GhCOR27 exhibited a more severe chilling injury phenotype than plants infiltrated with the vector control (Figure 4C). The percentage of survival plants silenced with GhCOR27 was also lower than those with control infiltration (Figure 4D). Thus, loss of function analysis suggested that GhCOR27 may play a role in cold stress signal pathway.

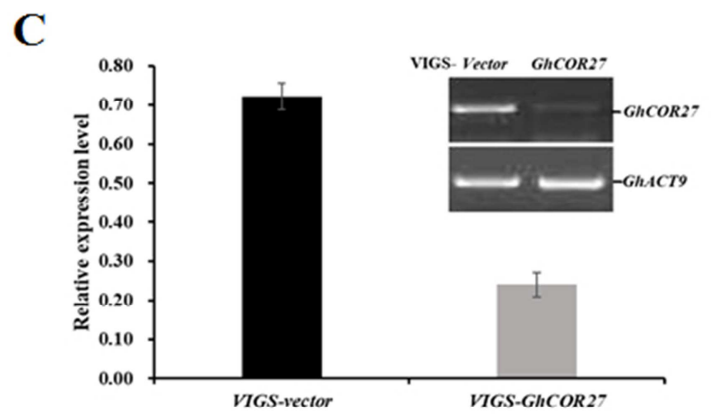

D

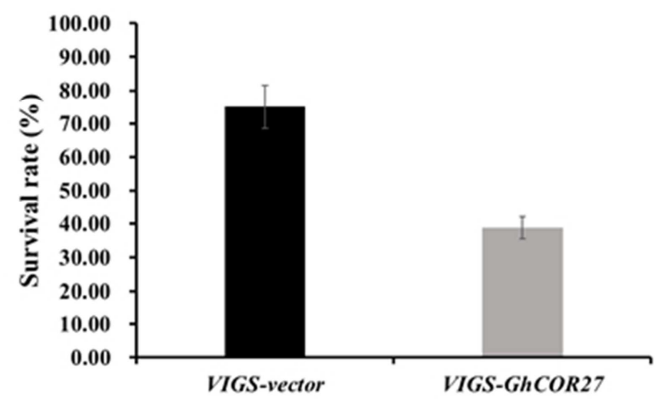

Figure 4. Silencing of GhCOR27 enhanced plant susceptibility to cold stress.

A. The photobleaching phenotype of GhCLA1-silenced cotton leaves. Agrobacterium carrying pTRV-RNA1 and pYL156-GhCLAl were infiltrated into two fully expanded cotyledons of 2-week-old seedlings. The right and the inset show the expression of GhCLA1 in control and silenced plants by RT-PCR and qRT-PCR analysis. GhACT9 was used as a control. B. Phenotype of control and silenced seedlings under low temperature condition. C. The relative expression of GhCOR27 in control and GhCOR27-silenced plants, the inset show the expression of GhCLA1 in control and silenced plants by RT-PCR. D. Survival rate of GhCOR27-silenced seedlings after low temperature treatment. 


\section{Discussion}

Many $C O R$ genes from different plants have been described, including five groups of $C O R$ genes from $A$. thaliana, $C b C O R 15$ from $C$. bungeana, BN115, BN26, and BN19 genes from B. napus, COR14b gene from Hordeum vulgare, and CbCOR15a from $C$. bursa-pastoris. These genes are of different expression patterns. For example, BnCOR25 from $B$. napus transcription level significantly increased after $3 \mathrm{~h}$ cold treatment, and declined to low level after $6 \mathrm{~h}$ and enhanced again after $12 \mathrm{~h}$ cold treatment [16, 17]. Different expression patterns may indicate different roles of these COR genes in each species, for example in Arabidopsis, COR27 and COR28 mediate cold signaling input to circadian clock and represent a trade-off between freezing tolerance and appropriate period length of circadian rhythm [11]. Cotton (Gossypium spp.) is the most important fiber producing plant in the world and also a significant oilseed crop. Due to cotton is thermophilic and sensitive to cold temperature during its development and growth, it is important to improve our understanding of genes that directly or indirectly impact such cold traits. Based on the whole cotton genome sequence database, a number of cotton genes were identified and characterized, the understanding of gene function not only at genome-wide level, but also at individual level becomes systematic. In this study, we used the full length AtCOR27 amino acid sequence as query to blast against the genome of $G$. hirsutum database and screened $C O R$ family member in the cotton genome. Total nine genes were identified from $G$. hirsutum database. Then, quantitative PCR was performed to find out which candidates were markedly up-regulated under cold stress. The expression level of GhCOR27 increased after $3 \mathrm{~h}$ cold treatment and then reached the peak that was 1.92 fold higher in the cold-treated seedlings than in those of the control plants, even treated after $48 \mathrm{~h}$, it remained a higher transcription level than non-treatment condition, which indicate that GhCOR27 may play an important role in response to cold stress.

For further understanding of the function of GhCOR27 under cold stress, we should have generated over-expressing or loss of function cotton plants to characterize it. As the major impediment to analyzing cotton gene function on a large scale is the laborious and time-consuming process of generating transgenic cotton. Moreover, many cotton cultivars and Gossypium species that contain important genes for cotton improvement are recalcitrant to genetic transformation. Therefore, there is an urgent need to develop a rapid method for functional analysis of cotton genes on a genomic scale. Virus-induced gene silencing (VIGS) offers such a possibility because it allows the investigation of gene functions without plant. By now, VIGS has been a reliable gene-silencing technique for studying gene characterization in plant. Furthermore, VIGS vectors have been modified to apply to different species and been already proved to work efficiently. Among these vectors, the tobacco rattle virus (TRV) vector is widely used in many plants such as tobacco [18], tomato [19], pepper [20] and petunia [21]. By this approach, genes referred to abiotic stress in cotton were identified and characterized as well $[14,15,22]$. More important, VIGS provides rapid way to discovery and study gene because when the Agrobacterium carrying the target gene is inoculated in cotton cotyledons of 2-week-old seedling, the effect of gene silencing will emerge in 2 weeks after the inoculation [20], which is especially helpful for those plants which have difficulty in obtaining transgenic plants. Silencing begins within a few weeks of inoculation and can extend throughout the developing period. VIGS can also be used to test the function of essential genes [23]. Gao found that GhMKK2 and GhVel are required for resistance to Verticillium dahliae in the cotton CA4002 line by VIGS method [20]. So, in this study we used the VIGS technology for the purpose of generating GhCOR27-silenced cotton plants. The comparison result indicated that silencing GhCOR27 led to plant susceptibility to cold stress which the survival rate of GhCOR27-silenced seedlings was only at $31.09 \%$ lower than those of non-silenced seedlings at $53.27 \%$. These results suggest that GhCOR27 may play a role in response to cotton cold stress response and demonstrate the TRV-VIGS system can be used to rapidly identify functions of genes that play a role in cotton abiotic stress.

\section{Acknowledgements}

This work was supported by the Key Laboratory Projects of Xinjiang, China (No. 2015KL008).

\section{References}

[1] Barrero-Gil, J. and J. Salinas (2013) Post-translational regulation of cold acclimation response. Plant science 205: $48-54$.

[2] Kargiotidou, A., et al. (2010) Cold acclimation and low temperature resistance in cotton: Gossypium hirsutum phospholipase $\mathrm{D} \alpha$ isoforms are differentially regulated by temperature and light. Journal of experimental botany 61 (11): 2991-3002.

[3] Thomashow, M. F. (1999) Plant cold acclimation: freezing tolerance genes and regulatory mechanisms. Annual review of plant biology 50 (1): 571-599.

[4] Zhu, X., et al. (2013) Aequorin-based luminescence imaging reveals stimulus-and tissue-specific $\mathrm{Ca} 2+$ dynamics in Arabidopsis plants. Molecular plant 6 (2): 444-455.

[5] Shi, Y., et al. (2014) Cold signal transduction and its interplay with phytohormones during cold acclimation. Plant and Cell Physiology 56 (1): 7-15.

[6] Chinnusamy, V., et al. (2006) Gene regulation during cold acclimation in plants. Physiologia Plantarum 126 (1): 52-61.

[7] An, D., et al. (2017) Cassava C-repeat binding factor 1 gene responds to low temperature and enhances cold tolerance when overexpressed in Arabidopsis and cassava. Plant molecular biology 94 (1-2): 109-124.

[8] Uemura, M., et al. (1996) Effects of COR6. 6 and COR15 am polypeptides encoded by COR (cold-regulated) genes of Arabidopsis thaliana on the freeze-induced fusion and leakage of liposomes. Plant Physiology 111 (1): 313-327. 
[9] Lee, H., et al. (1999) Cold-regulated gene expression and freezing tolerance in an Arabidopsis thaliana mutant. Plant Journal 17 (3): 301-308.

[10] Thomashow, M. F. (2001) So what's new in the field of plant cold acclimation? Lots! Plant Physiology 125 (1): 89-93.

[11] Wang, P., et al. (2017) COR27 and COR28 encode nighttime repressors integrating Arabidopsis circadian clock and cold response." Journal of integrative plant biology 59 (2): 78-85.

[12] Wang, D.-Z., et al. (2017) Gene regulation and signal transduction in the ICE-CBF-COR signaling pathway during cold stress in plants. Biochemistry (Moscow) 82 (10): 1103-1117.

[13] Gao, X., et al. (2011). Silencing GhNDR1 and GhMKK2 compromises cotton resistance to Verticillium wilt. Plant Journal 66 (2): 293-305.

[14] Gao, X. and L. Shan (2013) Functional genomic analysis of cotton genes with agrobacterium-mediated virus-induced gene silencing. Virus-Induced Gene Silencing, Springer: 157-165.

[15] Gu, Z., et al. (2014) A versatile system for functional analysis of genes and micro RNA $\mathrm{s}$ in cotton. Plant biotechnology journal 12 (5): 638-649.

[16] Si, J., et al. (2009) CbCOR15, a cold-regulated gene from alpine Chorispora bungeana, confers cold tolerance in transgenic tobacco. Journal of Plant Biology 52 (6): 593.

[17] Chen, L., et al. (2011) A novel cold-regulated gene, COR25, of Brassica napus is involved in plant response and tolerance to cold stress. Plant cell reports 30 (4): 463-471.
[18] Liu, Y., et al. (2004) Virus induced gene silencing of a DEFICIENS ortholog in Nicotiana benthamiana. Plant molecular biology 54 (5): 701-711.

[19] Peele, C., et al. (2001) Silencing of a meristematic gene using geminivirus-derived vectors. Plant Journal 27 (4): 357-366.

[20] Jordan, C. V., et al. (2007) Geminivirus-induced gene silencing of the tobacco retinoblastoma-related gene results in cell death and altered development. Plant molecular biology 65 (1-2): 163-175.

[21] Gao, X., et al. (2013) Cotton GhBAK1 Mediates Verticillium Wilt Resistance and Cell Death. Journal of integrative plant biology 55 (7): 586-596.

[22] Chung, E., et al. (2004) A method of high frequency virus induced gene silencing in chili pepper (Сарsісит аппиит L. cv. Bukang). Mol Cells 17 (2): 377-380.

[23] Donnelly, L. M., et al. (2010) Virus-induced gene silencing of a NAC transcription factor alters flower morphology and accelerates flower senescence in petunia. HortScience, AMER SOC HORTICULTURAL SCIENCE 113 S WEST ST, STE 200, ALEXANDRIA, VA 22314.

[24] Tuttle, J. R., et al. (2008) Geminivirus-mediated gene silencing from Cotton leaf crumple virus is enhanced by low temperature in cotton. Plant Physiology 148 (1): 41-50.

[25] Fu, D. Q., et al. (2005) Virus-induced gene silencing in tomato fruit. Plant Journal 43 (2): 299-308. 